\title{
Unusual Presentation of Primary Hyperparathyroidism: A Rare Case Report
}

\author{
${ }^{1}$ Veeramaneni S Teja, ${ }^{2}$ Ramya Ramakrishnan
}

\section{ABSTRACT}

Aim: The aim of this case report is to highlight an exceptional presentation of primary hyperparathyroidism.

Background: Asymptomatic maternal hyperparathyroidism manifesting in the neonate as hypocalcemic convulsions is exceedingly rare.

Case report: Primary hyperparathyroidism has got a varied presentations. We present a case report of neonatal hypocalcemic convulsions secondary to asymptomatic maternal hyperparathyroidism. Here, the challenge in diagnosing this condition and management will be discussed in the context of available literature.

Conclusion: In the event of nonfebrile seizures in the neonate due to hypocalcemia, the mother has to be evaluated for primary hyperparathyroidism.

Clinical significance: Physicians' awareness of this unique manifestation of primary hyperparathyroidism is essential for the appropriate management of both the mother and the neonate.

Timely identification and treatment of this condition will prevent complications in both.

Keywords: Neonatal hypocalcemia, Primary hyperparathyroidism, Seizures

How to cite this article: Teja VS, Ramakrishnan R. Unusual Presentation of Primary Hyperparathyroidism: A Rare Case Report. World J Endoc Surg 2017;9(1):13-15.

\section{Source of support: Nil}

Conflict of interest: None

\section{INTRODUCTION}

Primary hyperparathyroidism is an endocrine disorder with a variable clinical expression. The most common cause of primary hyperparathyroidism is a solitary adenoma. Around 95\% of patients are absolutely asymptomatic. Asymptomatic maternal primary hyperparathyroidism manifesting in the neonate as hypocalcemic

\footnotetext{
${ }^{1}$ Junior Resident, ${ }^{2}$ Professor

1,2Department of General Surgery, Sri Ramachandra Medical College and Research Institute, Chennai, Tamil Nadu, India

Corresponding Author: Ramya Ramakrishnan, Professor Department of General Surgery, Sri Ramachandra Medical College and Research Institute, Chennai, Tamil Nadu, India, Phone: +919884396099, e-mail: shravan_veeramaneni@yahoo.co.in
}

convulsions is exceedingly rare. Here, we present a case report of neonatal convulsions secondary to asymptomatic maternal primary hyperparathyroidism. Physicians should be aware of this presentation and thus, when neonates present with hypocalcemic seizures, selected investigations have to be done to establish or refute a diagnosis of maternal hyperparathyroidism.

\section{CASE REPORT}

A 26-year-old multiparous woman with no significant preexisting medical condition delivered a girl baby weighing $2600 \mathrm{gm}$ by spontaneous cephalic presentation at 38 weeks of gestation. The neonate, on day 3 of life, was brought to the emergency department with severe tonic-clonic convulsions and was admitted. Physical examination revealed an active, afebrile neonate with normal physical findings and facial appearance. Seizures were not controlled with the regular anticonvulsant drugs. Complete panel of routine blood and urine investigations and imaging was done and were all found to be normal. Later, thyroid profile, magnesium, calcium, phosphorous, and alkaline phosphatase were done and the baby was found to have hypocalcemia with calcium value of $5 \mathrm{mg} / \mathrm{dL}$. Baby's serum calcium levels reached normal level with IV calcium gluconate infusions. Convulsions were controlled and baby was put on oral calcium supplements at the time of discharge. Baby did not have any further episodes of seizures in early infant period and calcium levels returned back to normal by 4 months of age.

Suspecting a maternal etiology for the neonatal hypocalcemia, the baby's mother was investigated and serum calcium was found to be high with a value of $12 \mathrm{mg} / \mathrm{dL}$ (9-11mg/dL). Serum intact parathormone was grossly elevated to $364.60 \mathrm{pg} / \mathrm{dL}$ (10-60 pg/dL). Mother had neither complaints nor a history of major preexisting medical condition. Physical examination of the mother was normal. Renal function test was normal. Primary hyperparathyroidism was suspected and ultrasound neck was done which showed a $2 \times 0.8 \mathrm{~cm}$ hypoechoic lesion in the inferior aspect of right lobe of the thyroid, suggestive of parathyroid adenoma. Sestamibi scan was done, which showed persistent increased uptake in the right inferior parathyroid gland strongly suggestive of 


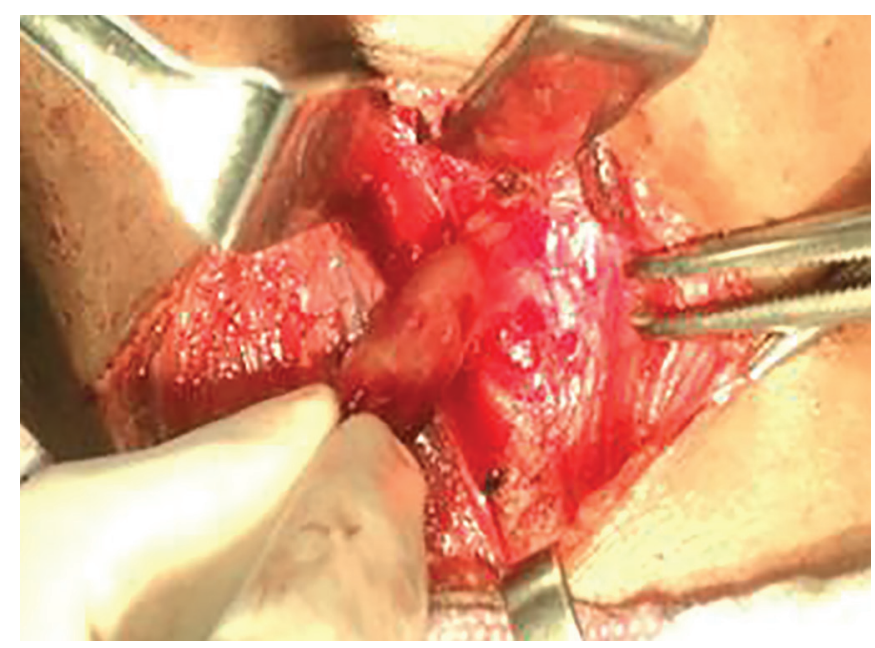

Fig. 1: Intraoperative view of the parathyroid gland

right inferior parathyroid adenoma. Patient was counselled and she underwent minimally invasive focused parathyroidectomy.

Under general anesthesia, unilateral $2 \mathrm{~cm}$ incision made on the right side of neck for a focused minimally invasive right parathyroidectomy, tumor identified and excised in toto (Fig. 1). Serum intraoperative parathormone (ioPTH) done 20 minutes after the removal of the gland showed a value of $17.7 \mathrm{pg} / \mathrm{dL}$ which reflects that the diseased gland is removed as there is more than $50 \%$ fall in ioPTH. Frozen section confirmed the tissue sent as parathyroid.

Postoperative period was uneventful. Daily serum calcium estimated for 5 days which showed an initial rise of calcium followed by gradual normalization of the level (Graph 1). Follow-up after 1 week for suture removal with repeat calcium was normal and wound healing was perfect. Histopathology was reported as benign parathyroid adenoma.

\section{DISCUSSION}

The most common cause of primary hyperparathyroidism is a solitary adenoma which accounts for $80 \%$. Hyperplasia accounts for $15 \%$ and parathyroid carcinoma for $1 \%{ }^{1}$ The classical presentation of primary hyperparathyroidism presents with a pentad of symptoms, i.e., renal stones, painful bones, abdominal groans, psychic moans, and overtones has now become exceedingly rare. Asymptomatic primary hyperparathyroidism can lead to complications like nephrolithiasis, pancreatitis, gastrointestinal ulcers, and endangering hypercalcemic crisis. ${ }^{1}$ The typical biochemical abnormalities seen in these patients are hypercalcemia, hypophosphatemia, elevated serum parathormone, and alkaline phosphatase levels. ${ }^{2}$ Sestamibi scan is the gold standard for the diagnosis of parathyroid adenoma.

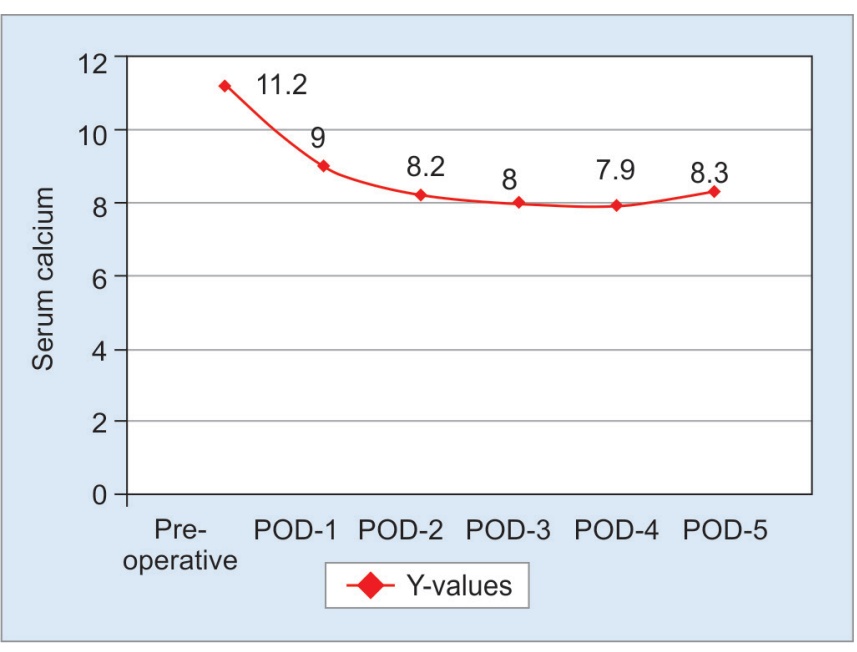

Graph 1: Serial serum calcium levels (POD: Postoperative day)

In pregnant patients, the symptoms of hyperparathyroidism are usually fatigue, thirst, constipation, or depression, and all these are mostly attributed to the pregnancy and hence, the diagnosis can be easily missed. ${ }^{3}$ In the fetus, intrauterine growth retardation, low birth weight, preterm delivery, neonatal hypocalcemic tetany, seizures, and intrauterine death may result as a consequence of unrecognized maternal hyperparathyroidism. ${ }^{4}$ In neonates, transient early hypocalcemia is common which resolves spontaneously.

Primary hyperparathyroidism in the mother suppresses the parathyroid gland in the fetus. As maternal parathormone crosses the placenta and results in impaired calcium homeostasis after birth leading on to neonatal hypocalcemia. ${ }^{5}$ Neonatal hypocalcemia due to maternal hyperparathyroidism was first described by Friedrichsen in 1938. He classified this entity into early onset ( $<48$ hours of life) and late onset (1st week of life). ${ }^{6}$ Hypocalcemia in neonate resulting from primary hyperparathyroidism in mother is usually found in the first 2 weeks of life. Neonates usually present with poor feeding, hypotonia, stridor, and irritability and the most striking one, are the convulsions. Cardiac involvement is lethal which may manifest as tachycardia, heart failure, prolonged QT interval, or decreased contractility ${ }^{7}$

Hypocalcemic seizures in neonates usually are of late onset, ${ }^{8}$ contrary to which our case presented with early onset convulsions. Neonatal convulsions not controlled by conventional anticonvulsants should raise the possibility of a metabolic cause - namely, hypocalcemia secondary to maternal primary hyperparathyroidism. ${ }^{9}$ Hence, high index of suspicion is required to identify maternal asymptomatic primary hyperparathyroidism.

Minimally invasive unilateral approaches to the parathyroid are becoming very popular. ${ }^{6}$ Unlike the traditional approach, where all four parathyroid glands 
are exposed, here we used a novel method of minimally invasive focused parathyroidectomy as the procedure of choice. This involves smaller incision, minimal dissection, and esthetically acceptable scar. In our case, biochemical frozen section was also done to confirm the removal of the diseased gland, where intraoperative assay was done 20 minutes after the removal of the diseased gland and there was more than $50 \%$ fall in the parathormone levels.

\section{CONCLUSION}

Unexplained nonfebrile seizures in a neonate have to be evaluated biochemically. If hypocalcemia is found to be the cause of the convulsions, the mother has to be evaluated for primary hyperparathyroidism. Sestamibi scan should be used as a gold standard imaging to localize the pathology and minimally invasive focused parathyroidectomy has become established as a viable surgical option to cure the patient.

\section{CLINICAL SIGNIFICANCE}

Physicians should be aware of this presentation and thus, when neonates present with hypocalcemic seizures, selected investigations have to be done and maternal hyperparathyroidism has to be ruled out.

Complications in both mother and fetus secondary to asymptomatic primary hyperparathyroidism can be prevented and morbidity is reduced.

\section{REFERENCES}

1. Beattie GC, Ravi NR, Lewis M, Williams H, Blair AW, Campbell IW, Browning GGP. Rare presentation of maternal primary hyperparathyroidism. BMJ 2000 Jul 22;321(7255): 223-224

2. Ip P. Neonatal convulsion revealing maternal hyperparathyroidism: an unusual case of late neonatal hypoparathyroidism. Arch Gynecol Obstet 2003 Aug;268(3):227-229.

3. Cherry TA, Kauffman RP, Myles TD. Primary hyperparathyroidism, hypercalcemic crisis and subsequent seizures occurring during pregnancy: a case report. J Maternal Fetal Neonatal Med 2002 Nov;12(5):349-352.

4. Schnatz PF, Curry SL. Primary hyperparathyroidism in pregnancy: evidence-based management. Obstet Gynecol Surv 2002 Jun;57(6):365-376.

5. Jain A, Agarwal R, Sankar MJ, Deorari AK, Paul VK. Hypocalcemia in the newborn. Indian J Pediatr 2008 Feb;75(2):165-169.

6. Zachariah SK, Thomas PA. Primary hyperparathyroidism: a report of two unusual cases. Indian J Surg 2010 Apr;72(2): 135-137.

7. Poomthavorn P, Ongphiphadhanakul B, Mahachoklertwattana P. Transient neonatal hypoparathyroidism in two siblings unmasking maternal normocalcemic hyperparathyroidism. Eur JPediatr 2008 Apr;167(4):431-434.

8. Pieringer $\mathrm{H}$, Hatzl-Griesenhofer $\mathrm{M}$, Shebl $\mathrm{O}$, WiesingerEidenberger G, Maschek W, Biesenbach G. Hypocalcemic tetany in the newborn as a manifestation of unrecognized maternal primary hyperparathyroidism. Wien Klin Wochenschr 2007;119(3-4):129-131.

9. Brisse F, Breton D, Gagey V, Chéron G. Convulsions and neonatal hypoparathyrodi revealing hyperparathyroidism. Arch Pediatr 1994 Mar 1;(3):255-259. 